\title{
On the crucial cluster Andrews-Lindsay 1 and a $4 \%$ distance solution for its planetary nebula ${ }^{\star}$
}

\author{
D. Majaess ${ }^{1,2}$, G. Carraro ${ }^{3}$, C. Moni Bidin ${ }^{4}$, C. Bonatto ${ }^{5}$, D. Turner ${ }^{1}$, M. Moyano ${ }^{4}$, L. Berdnikov $^{6,7}$, and E. Giorgi ${ }^{8}$ \\ 1 Department of Astronomy \& Physics, Saint Mary's University, Halifax, NS B3H 3C3, Canada \\ e-mail: dmajaess@ap.smu.ca \\ 2 Mount Saint Vincent University, Halifax, NS B3M 2J6, Canada \\ 3 European Southern Observatory, Av. Alonso de Cordova 3107, Casilla 19001, Santiago, Chile \\ ${ }^{4}$ Instituto de Astronomía, Universidad Católica del Norte, Av. Angamos 0610, Antofagasta, Chile \\ 5 Departamento de Astronomia, Universidade Federal do Rio Grande do Sul, Av. Bento Gonalves 9500, 91501-970 Porto Alegre, \\ RS, Brazil \\ 6 Sternberg Astronomical Institute, Moscow M. V. Lomonosov State University, 119992 Moscow, Russia \\ 7 Isaac Newton Institute of Chile, Moscow Branch, Universitetskij Pr. 13, 119992 Moscow, Russia \\ 8 Facultad de Ciencias Astronómicas y Geofísicas (UNLP), Instituto de Astrofísica de La Plata (CONICET, UNLP), \\ Paseo del Bosque s/n, La Plata, Argentina
}

Received 19 February 2014 / Accepted 22 April 2014

\section{ABSTRACT}

\begin{abstract}
Andrews-Lindsay 1 is a pertinent open cluster because it may host the planetary nebula (PN) PHR 1315-6555, yet ambiguities linger concerning its fundamental parameters ( $>50 \%$ scatter). New multiband $B V J H W_{1-4}$ photometry for cluster and field stars, in concert with observations of recently discovered classical Cepheids, were used to constrain the reddening and velocity-distance profiles along the sightline. That analysis yielded the following parameters for the cluster: $E(J-H)=0.24 \pm 0.03, d=10.0 \pm 0.4 \mathrm{kpc}$ $\left(d_{J H}=9.9 \pm 0.6 \mathrm{kpc}, d_{B V}=10.1 \pm 0.5 \mathrm{kpc}\right)$, and $\log \tau=8.90 \pm 0.15$. The steep velocity-distance gradient along $\ell \sim 305^{\circ}$ indicates that two remote objects sharing spatial and kinematic parameters (i.e., PHR 1315-6555 and Andrews-Lindsay 1) are associated, thus confirming claims that the PN is a cluster member. The new distance for PHR 1315-6555 is among the most precise established yet for a Galactic PN $(\sigma / d=4 \%)$.
\end{abstract}

Key words. open clusters and associations: individual: Andrews-Lindsay 1 (ESO 96-SC04) planetary nebulae: individual: PHR 1315-6555

\section{Introduction}

The motivation behind the search for planetary nebulae $(\mathrm{PNe})$ in star clusters is to secure precise parameters for the nebulae. Those parameters include the PN's distance, physical dimensions, age, chemical composition, and potentially progenitor mass (Parker et al. 2011; Turner et al. 2011; Moni Bidin et al. 2014). A solid PN distance could likewise be used to calibrate relationships aimed at establishing distances to field $\mathrm{PNe}$ (Frew 2008). Reliable distances are particularly desirable since precise parallaxes exist for a mere fraction of the nearest $\mathrm{PNe}$ (Benedict et al. 2009, $\sigma / \pi \sim 5 \%$ ), whereas distance estimates for the bulk of PNe display $20 \%$ to $30 \%$ uncertainties or larger (Stanghellini et al. 2008; Giammanco et al. 2011, the latter's Fig. 6). Admittedly, the quest to identify numerous cluster PNe has been hampered by several factors. Massive PNe associated with younger clusters feature short lifetimes $\left(10^{3}-10^{4}\right.$ years $)$. The matter is compounded by the paucity of old clusters that spawn lower mass PNe exhibiting longer lifetimes. Star clusters rarely survive beyond $10^{7}$ years (Bonatto \& Bica 2011), and the majority that do surpass that threshold host evolved constituents that terminate as SNe (e.g., Majaess et al. 2007, their Fig. 2). Furthermore, the bulk of cataloged Galactic PNe are members of

* Calibrated photometry is only available at the CDS via anonymous ftp to cdsarc.u-strasbg. fr (130.79.128.5) or via http://cdsarc.u-strasbg.fr/viz-bin/qcat?]/A+A/567/A1 the bulge (Majaess et al. 2007, their Fig. 1), which underscores the pertinence of surveys aimed at discovering PNe throughout the Galactic disk where younger clusters reside (Parker et al. 2006; the Macquarie/AAO/Strasbourg $\mathrm{H} \alpha$ PN Catalogue, see also Jacoby et al. 2010; Lutz et al. 2012).

Consequently, few promising cases of PNe in Galactic clusters have been reported, and thus any bona fide pairs ${ }^{1}$ are crucial for various research topics (e.g., constraining the impact of mass loss). The search for extragalactic cluster PNe has yielded similar results. Jacoby et al. (2013) observed 467 star clusters in M 31 to detect PNe that share the former's velocity. That evaluation enables chance superpositions to be identified, in addition to contamination from unrelated emission sources along the sightline (e.g., H II regions). Jacoby et al. (2013) concluded that five (of 270) globular clusters may host PNe, whereas those targets identified near open clusters likely constitute chance alignments.

A subsample of the tentative Galactic PNe/open cluster pairs includes Abell 8 and Bica 6 (Bonatto et al. 2008; Turner et al. 2011), He 2-86 and NGC 4463 (Majaess et al. 2007; Moni Bidin et al. 2014), and PHR 1315-6555 and Andrews-Lindsay 1 (Parker et al. 2006, 2011; Majaess et al. 2007; Frew 2008) ${ }^{2}$.

\footnotetext{
Perhaps an equally important endeavour is to eliminate unreliable calibrators and reputed associations (Majaess et al. 2007; Kiss et al. 2008; Moni Bidin et al. 2014).

2 PHR 1315-6555 is likewise designated as PNG 305.3-03.1, and Andrews-Lindsay 1 is cataloged as ESO 96-SC04 and VdB 144.
} 
Table 1. Parameters for Andrews-Lindsay 1 (AL1).

\begin{tabular}{lcllllc}
\hline \hline Reference & Target & $d(\mathrm{kpc})$ & $E(B-V)$ & $\tau(\mathrm{Gyr})$ & $\mathrm{RV}\left(\mathrm{km} \mathrm{s}^{-1}\right)$ & {$[\mathrm{Fe} / \mathrm{H}]$} \\
\hline Janes \& Phelps (1994) & AL1 & 7.57 & 0.72 & $\ldots$ & $\ldots$ & $\ldots$ \\
Carraro et al. (1995) & AL1 & 11.8 & 0.75 & 0.7 & $\ldots$ & subsolar \\
Carraro \& Munari (2004) & AL1 & $12 \pm 1$ & $0.7 \pm 0.2$ & 0.8 & $\ldots$ & $\ldots$ \\
Frinchaboy et al. (2004a) & AL1 & $\ldots$ & $\ldots$ & $\ldots$ & $40 \pm 10$ & $-0.51 \pm 0.30$ \\
Frinchaboy et al. (2004b) & AL1 & 9.35 & $\ldots$ & 0.67 & $\ldots$ & $\ldots$ \\
Carraro et al. (2005) & AL1 & $16.95^{b}$ & $0.34 \pm 0.05$ & $0.8 \pm 0.2$ & $\ldots$ & $\ldots$ \\
Frew (2008) & PN & $9.7 \pm 3.1$ & $0.71^{a}$ & $\ldots$ & $51.6 \pm 15.0$ & $\ldots$ \\
& AL1 & $\ldots$ & $\ldots$ & $\ldots$ & $50 \pm 10$ & $\ldots$ \\
Parker et al. (2011) & AL1 & $\ldots$ & $\ldots$ & $\ldots$ & $57 \pm 5^{a}$ & $\ldots$ \\
& PN & $10.4 \pm 3.4$ & $0.83 \pm 0.08$ & $\ldots$ & $58 \pm 2.5$ & $\ldots$ \\
& & & & & $59 \pm 2$ & \\
\hline
\end{tabular}

Notes. ${ }^{(a)}$ See text. ${ }^{(b)}$ Observations were undertaken during unsatisfactory weather conditions.

Yet another important consideration arises when inferring the progenitor mass of those PNe from single stars near the cluster turnoff. The canonical hypothesis advocating that PNe stem from single stars does not readily explain their non-spherical morphologies or low formation rate (Jacoby et al. 2013, see also De Marco et al. 2013). Indeed, the detection of PNe within globular clusters, which exhibit turnoff masses below the $1 M_{\odot}$ threshold predicted by $\mathrm{PNe}$ models, implies that the progenitor may have been augmented by mass transfer. Specifically, Jacoby et al. (2013) noted that four (of 130) Galactic globular clusters host PNe, with the cases split between PNe featuring non-spherical nebulae and high-mass central stars conducive to younger clusters. That evidence, in concert with the realization that three PNe are located in globular clusters hosting numerous X-ray sources, supports claims that multiplicity affects the formation of globular cluster PNe.

In each of these Galactic cases further independent research is required, with a validation standard on par with the magnitude of the discovery. For example, velocities measured for the PN NGC 2438 by Pauls \& Kohoutek (1996) indicated it was a cluster member (M 46), whereas those by O'dell (1963) suggested otherwise. Independent observations urged by Majaess et al. (2007) and others were subsequently published by Kiss et al. (2008), and they suggest that the pair constitute a chance alignment along the sightline. Radial velocities for Abell 8 and Bica 6 likewise require confirmation, especially given their potential implications for Galactic dynamics (Turner 2013). NGC 4463 may host a rare young PN (He 2-86) that exhibits sizable internal extinction (Moni Bidin et al. 2014, their comprehensive discussion in Sect. 5), and the cluster is pertinent for stellar evolution research. Specifically, NGC 4463 may host a F-supergiant (post onset of core helium burning), the PN, and a massive blue straggler (Ahumada \& Lapasset 2007).

Continuing the cluster PNe project spearheaded by Moni Bidin et al. (2014), this study aims to bolster the link tying PHR 1315-6555 to Andrews-Lindsay 1 by resolving the faint cluster's presently ambiguous fundamental parameters (Table 1). New $B V J H W_{1-4}$ observations were analyzed to achieve that objective. $W_{1-4}$ are the four WISE mid-infrared passbands (Wright et al. 2010).

\section{Analysis}

\subsection{Prior studies of AL1}

Distance estimates cited for Andrews-Lindsay 1 span a factor of two (7.57-16.95 kpc, Janes \& Phelps 1994; Carraro et al. 2005).

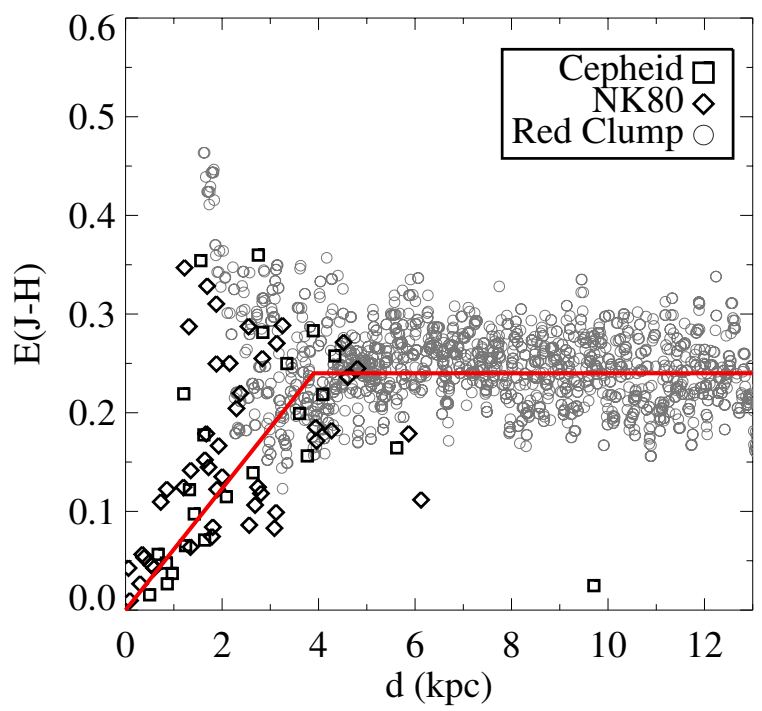

Fig. 1. Reddening-distance profile for the sightline encompassing Andrews-Lindsay 1. Classical Cepheids (Berdnikov et al. 2000; Berdnikov 2008) and results from Neckel et al. (1980, NK80) were used to trace the local region, while red clump giants were employed to extend the distance baseline. The mean color excess for remote sources $\left(E_{J-H}=0.24 \pm 0.03 \rightarrow E_{B-V} \sim 0.72\right)$ agrees with estimates from optical analyses of Andrews-Lindsay 1, to within the uncertainties (Table 1).

A similar spread exists for the extinction $\left(E_{B-V}=0.35-0.75\right)$. The ambiguity arises from the challenging nature of assessing a distant, faint, and reddened cluster that is superposed upon a field that introduces significant contamination (Carraro et al. 1995, their Fig. 1). However, a consensus exists concerning the cluster age ( $\tau \sim 0.8 \mathrm{Gyr})$, which was partly inferred from the relative positions of the cluster's red clump and blue turnoff stars.

The first comprehensive photometric analysis of the cluster was probably undertaken by Janes \& Phelps (1994), who obtained multiband $B V I_{\mathrm{c}}$ observations from the CTIO 0.9-m telescope. The cluster parameters established were $d=7.57 \mathrm{kpc}$ and $E(B-V)=0.72$, as deduced in part from an inspection of the red clump demographic. Carraro et al. (1995) obtained deeper $B V$ photometry from La Silla that fostered an improved assessment and yielded $d \sim 11.8 \mathrm{kpc}, E(B-V) \sim 0.75$, and $\tau \sim 0.7$ Gyr. Carraro et al. (1995) further remarked that cluster stars may exhibit subsolar abundances, a suggestion confirmed by subsequent abundance measurements determined using the Blanco 4-m telescope $([\mathrm{Fe} / \mathrm{H}]=-0.51 \pm 0.30$, Frinchaboy et al. 2004a). 
Carraro \& Munari (2004) expanded the Carraro et al. (1995) analysis by acquiring SAAO $I_{\mathrm{c}}$ observations, along with new $B V$ photometry. Those SAAO data are independent of the photometric zero point established by Janes \& Phelps (1994). The Janes \& Phelps (1994) observations were used as local standards by Carraro et al. (1995). An independent evaluation is important since Carraro \& Munari (2004) discovered a sizable offset relative to the Janes \& Phelps (1994) photometry: $\Delta(B-V)=$ $-0.053 \pm 0.080$ and $\Delta V=-0.098 \pm 0.049$. Carraro \& Munari (2004) derived the cluster reddening via a $(B-V)$ vs. $\left(V-I_{\mathrm{c}}\right)$ color-color analysis (Munari \& Carraro 1996), and concluded that Andrews-Lindsay 1 is characterized by $E(B-V)=0.7 \pm 0.2$, $d=12 \pm 1 \mathrm{kpc}$, and $\tau=800 \mathrm{Myr}$. Generally, prior estimates cited in the literature did not feature uncertainties and were typically deemed approximate. Frinchaboy et al. (2004a) obtained a radial velocity estimate from two cluster stars $(R V=40 \pm$ $10 \mathrm{~km} \mathrm{~s}^{-1}$ ), and Frinchaboy et al. (2004b) cite general parameters for Andrews-Lindsay 1 of $d=9.35 \mathrm{kpc}$ and $\tau=0.67 \mathrm{Gyr}$ (their Table 1). Conversely, Carraro et al. (2005) acquired new CTIO $B V I_{\mathrm{c}}$ photometry and deduced $d=16.95 \mathrm{kpc}, E(B-V)=$ $0.34 \pm 0.05$, and $\tau=800 \pm 200 \mathrm{Myr}$ (their Table 4). An underestimated $E(B-V)$ can result in an overestimated distance.

Frew (2008) has analyzed SAAO observations and discovered that the PN and cluster velocities are comparable, namely $51.6 \pm 15.0 \mathrm{~km} \mathrm{~s}^{-1}$ and $50 \pm 10 \mathrm{~km} \mathrm{~s}^{-1}$ accordingly. Moreover, Frew (2008) used the $H \alpha$ SB-r relation to determine a distance for PHR 1315-6555 ( $d=9.7 \pm 3.1 \mathrm{kpc}, \sigma / d \sim 30 \%)$, which is consistent with estimates for the cluster, e.g., by Janes \& Phelps $(1994, d=7.54 \mathrm{kpc})$ and Carraro \& Munari $(2004, d=$ $12 \pm 1 \mathrm{kpc}$ ). Parker et al. (2011) bolstered the Frew (2008) findings by acquiring AAOmega data, which imply velocities for the $\mathrm{PN}$ of $58.0 \pm 2.5 \mathrm{~km} \mathrm{~s}^{-1}$ (six Paschen lines) and $59 \pm 2 \mathrm{~km} \mathrm{~s}^{-1}(\mathrm{H} \beta$ and [OIII]). A coauthor (Frinchaboy) on the Parker et al. (2011) study determined that three cluster stars exhibit a mean velocity of $57 \pm 5 \mathrm{~km} \mathrm{~s}^{-1}$, which presumably supersedes the earlier Frinchaboy et al. (2004a) estimate (Table 1). Parker et al. (2011) note that the preliminary SAAO data discussed by Frew (2008) indicate a PN reddened by $E(B-V)=0.71$, while new highquality ANU data increase the result to $E(B-V)=0.83 \pm 0.08$. The latter was paired with other parameters to establish a revised $H \alpha$ SB-r PN distance of $d=10.4 \pm 3.4 \mathrm{kpc}$, which is marginally greater than the initial Frew (2008) estimate. Parker et al. (2011) conclude that consistent distances, velocities, and extinction estimates for the PN and (mean) cluster indicate that the two are associated. However, an independent assessment of the cluster is desirable given the scatter tied to the estimated distance and color excess. To that end Parker et al. (2011) examined $J H K_{\mathrm{S}}$ photometry from 2MASS, but concluded that the bulk of the cluster lies beyond the survey limits (Fig. 2). Furthermore, it has been pointed out that faint 2MASS photometry could be contaminated owing to the cluster's compact nature (e.g., Majaess et al. 2012a,b). In the following section the fundamental parameters of the cluster are resolved using deeper high-resolution photometry.

\subsection{Extinction and distance}

The first step in securing the cluster distance was to determine the reddening along $\ell \sim 305^{\circ}$, particularly given the spread among existing estimates. The new near-infrared data acquired for Andrews-Lindsay 1 contain numerous red clump giants, which enable the run of reddening to be established across a sizable distance baseline. Those observations were obtained via the Osiris instrument on the SOAR 4-m telescope (Cerro Pachón,

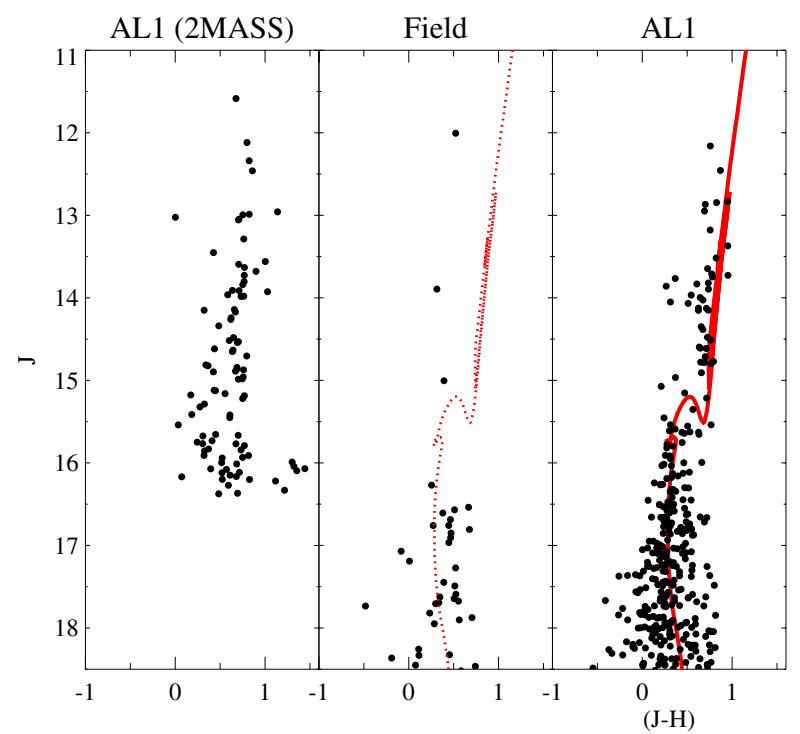

Fig. 2. Near-infrared color-magnitude diagrams for AndrewsLindsay 1. Fundamental parameters established for the cluster from the new data (right panel) are $d=9.9 \pm 0.6 \mathrm{kpc}$ and $\log \tau=8.90 \pm 0.15$.

Chile). Standard IRAF and DAOPHOT (Stetson 1987) routines were employed to extract the photometry. The instrumental photometry was subsequently standardized using comparatively uncrowded 2MASS stars in the broader field.

Red field stars were isolated in the near-infrared color-magnitude diagram ( $J$ vs. $J-H$ ), and assigned an absolute magnitude and intrinsic color tied to a red clump giant (Majaess et al. 2011a). Mean intrinsic parameters for a red clump star were inferred by pairing revised HIPPARCos parallaxes (van Leeuwen 2007) with the Skiff (2013) catalog of spectral classifications (Majaess et al. 2011a). A distance was computed using the standard extinction law, as deduced by Majaess et al. (in prep.) from a mapping of the broader region encompassing $\ell \sim 305^{\circ}$. That analysis relies on WISE midinfrared data (Wright et al. 2010) to determine extinction law variations via the color-extrapolation method. For comparison, certain regions in Carina and the Galactic bulge adhere to an anomalous extinction law (Pietrukowicz et al. 2012; Carraro et al. 2013).

The binned results of $\sim 1800$ potential red clump stars surrounding Andrews-Lindsay 1 are shown as Fig. 1. The findings were supplemented with data from Neckel et al. (1980), who delineated the run of reddening with distance for numerous Galactic sightlines using MK spectra and $\mathrm{H} \beta$ observations. The color excess and distance inferred from classical Cepheids occupying the broader region were likewise added to Fig. 1 (Berdnikov et al. 2000; Berdnikov 2008, and unpublished observations). The Cepheid distances were established via the latest Galactic Wesenheit $V I_{\mathrm{c}}$ function (Majaess et al. 2013), which relies partly on the HST parallaxes of Benedict et al. (2007) and the cluster Cepheids tabulated by Turner (2010) (see also Ngeow et al. 2014). The $V I_{\mathrm{c}}$ function was utilized owing to its diminished sensitivity to abundance variations (Majaess et al. 2011b). The color excess was computed with the period-color relation from Majaess et al. (2009) (see also Turner 2001).

The results of Fig. 1 imply that the color excess increases in tandem with distance to $d \sim 4 \mathrm{kpc}$ (see also Fig. 5), whereupon the trend becomes nearly constant. An inspection of the local spiral map (Majaess et al. 2011a, their Fig. 2) indicates that the Sagittarius-Carina arm is a source of extinction. The mean 


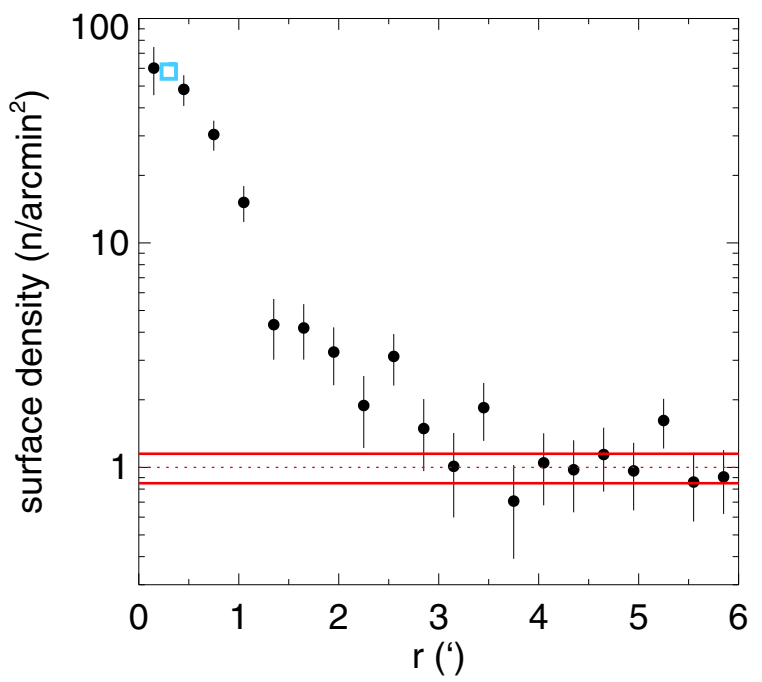

Fig. 3. Radial profile constructed from new $B V$ photometry for the region encompassing Andrews-Lindsay 1. The PN PHR 1315-6555 (blue square) lies well within the bounds of the cluster, which exhibits a corona that extends beyond $r \sim 2^{\prime}$.

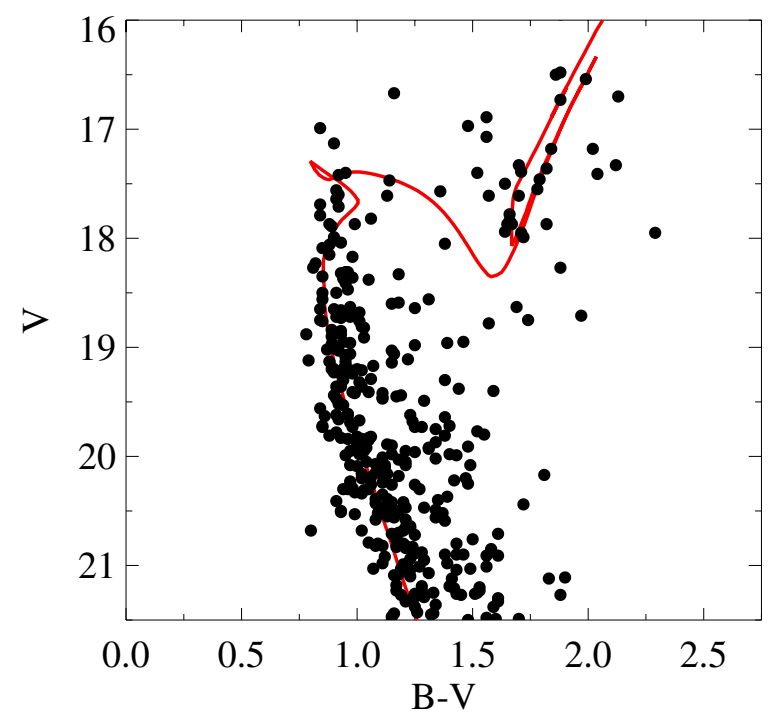

Fig. 4. $B V$ color-magnitude diagram of Andrews-Lindsay 1. A cluster distance of $d=10.1 \pm 0.5 \mathrm{kpc}$ is obtained, which supports the nearinfrared solution (Fig. 2). The observations are standardized to new photometry acquired for the field from the CTIO 1-m telescope.

reddening is $E(J-H)=0.24 \pm 0.03$, and that determination will help constrain the distance and age of Andrews-Lindsay 1. The corresponding optical reddening is $E(B-V) \sim 0.72$, which agrees with the cluster and PN color excess advocated by Carraro \& Munari (2004) and Parker et al. (2011), respectively (Table 1). The Schlafly \& Finkbeiner (2011) recalibration of the SFD dust maps yields $E(J-H) \sim 0.27$ for the sightline ${ }^{3}$.

The cluster distance may be ascertained by shifting an isochrone along the ordinate of the $(\mathrm{JH})$ color-magnitude diagram (Fig. 2), given that the color excess has been constrained above. A Padova isochrone (Girardi et al. 2002; Bonatto et al. 2004) was overlaid on the data with the aim of matching evolved blue and red stars, in concert with the upper most main sequence. Stars near the cluster core were examined to mitigate field contamination (Fig. 5, and see also Fig. 1 in Carraro et al. 1995).

\footnotetext{
3 NED extinction calculator: http://ned.ipac.caltech.edu/
}

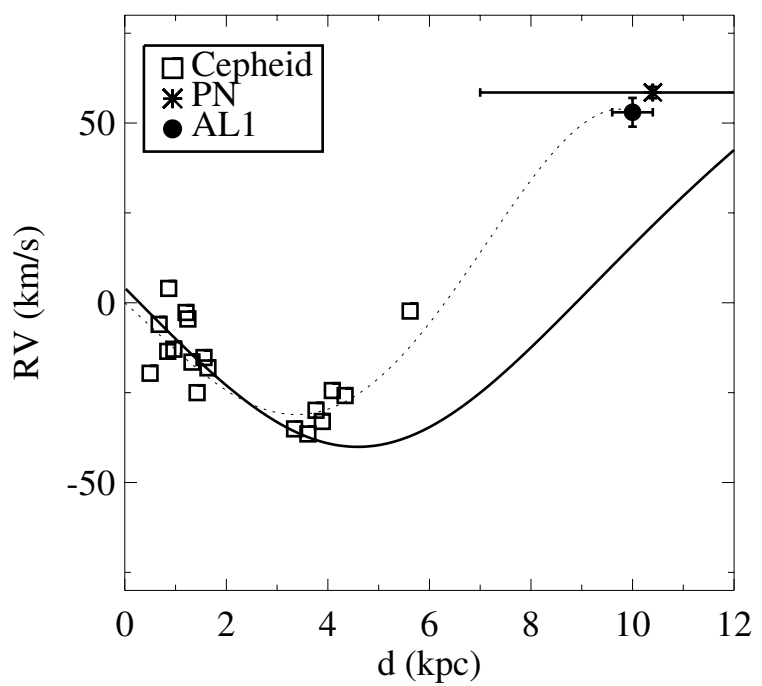

Fig. 5. Velocity-distance profile for the region encompassing Andrews-Lindsay 1. The trend predicted from a simple model for Galactic rotation is denoted by the solid line, whereas Cepheids were used to delineate the empirical profile (e.g., Berdnikov et al. 2000). The predicted relation is generally consistent with the observations, where nearer objects exhibit negative velocities and distant targets feature positive velocities. The spatial and kinematic agreement between the remote PN PHR 1315-6555 and the cluster Andrews-Lindsay 1 imply the former is probably a cluster member.

The fit implies a distance of $d=9.9 \pm 0.6 \mathrm{kpc}$ and an age of $\log \tau=8.90 \pm 0.15$. A comparison field presented in Fig. 2 does not follow the isochrone. A field-star decontamination algorithm (Bonatto \& Bica 2007, and references therein) that was applied corroborates the results, whereby the morphology displayed in the color-magnitude diagram by a field sample was removed from a diagram featuring field and cluster stars. Metallicity effects in the near-infrared are not acute (Alonso et al. 1996; Majaess et al. 2011c), and thus a solar isochrone was adopted. The Padova isochrone was adjusted to match the Majaess et al. (2011c) distance scale, which is not linked to the HIPPARcos results for the Pleiades, but rather to seven other comparatively nearby benchmark clusters that exhibit consistent $J H K_{\mathrm{s}}$ and revised HIPPARCos distances (van Leeuwen 2009; Majaess et al. 2011c). The Hyades and Praesepe are two of those clusters, and they share a similar age with Andrews-Lindsay 1. The intrinsic $J H K_{\mathrm{S}}$ relations are tied to revised HIPPARCOS parallaxes for stars within $d_{\odot}=25$ pc (van Leeuwen 2007); however, the principle concern promulgating the literature pertains to HIPPARCOS parallaxes for certain further targets (e.g., the Pleiades, Blanco 1, AQ Pup, Majaess et al. 2011c; Turner et al. 2012, see also van Leeuwen 2009, 2013 and discussion therein). The new cluster parameters constrain the PN's progenitor mass $\left(2.3 M_{\odot}\right)$, and they were paired with observations of the PN (Parker et al. 2011, their Table 5) to deduce its radius $(0.3 \mathrm{pc})$. That progenitor mass assumes single star evolution, which may be presumptuous for PNe (Jacoby et al. 2013; De Marco et al. 2013). An expansion velocity and observations related to the central star $(\mathrm{PN})$ are needed to glean further pertinent information.

New $U B V I_{\mathrm{c}}$ observations were acquired via the Y4CAM on the CTIO 1-m telescope to delineate the cluster's radial profile and check the infrared distance. Photometry was extracted using DAOPHOT and subsequently standardized to 46 stars in the Landolt (1992) SA 98 and PG 1047 fields. The new Y4CAM photometry span a sizable field of view, which fosters a reliable determination of the cluster's apparent size. The region 
occupied by cluster stars in the color-magnitude diagram was isolated to mitigate field contamination, and to improve the robustness of that determination. The resulting binned analysis implies that Andrews-Lindsay 1 features a corona that extends beyond $r \sim 2^{\prime}$ (Fig. 3). PHR 1315-6555 is $r \sim 23^{\prime \prime}$ from the center of Andrews-Lindsay 1, which in tandem with the suite of evidence presented thus far, is a further indication that the PN is associated with the cluster.

The new Y4CAM observations were employed to recalibrate the deeper $B V$ photometry of Carraro et al. (1995), which were originally tied to the Janes \& Phelps (1994) zero point. The reddening cited earlier was used to constrain an optical-based distance $(d=10.1 \pm 0.5 \mathrm{kpc}$, Fig. 4$)$. The result agrees with the near-infrared solution, and a weighted mean yields a distance of $d=10.0 \pm 0.4 \mathrm{kpc}$. That estimate implies that PHR 1315-6555 features among the most precise distances for PNe (4\%, see also Benedict et al. 2009).

The parameters were determined by matching the observed and intrinsic data via the traditional visual approach (e.g., Carraro \& Munari 2004; Bonatto \& Bica 2010), whereby the uncertainty represents the limit where a mismatch is clearly perceived. The traditional approach is subjective, and thus a complementary analysis was undertaken using the Bonatto (in prep.) computer algorithm for fitting isochrones (multidimensional minimization). That code yielded a consistent solution of $d=10.20 \pm 0.52 \mathrm{kpc}, E(B-V)=0.68 \pm 0.04$, and $\tau=810 \pm 100 \mathrm{Myr}$ (for $Z=0.019$ ). Paunzen \& Netopil (2006) note that errors tied to isochrone fitting via computer algorithms are comparable to those associated with the canonical approach, which appears supported by the present analysis.

A simplistic Galactic rotation model was employed to predict the velocity-distance profile for $\ell=305^{\circ}$, and the results are shown as Fig. 5. Andrews-Lindsay 1, PHR 1315-6555, and several Cepheids adhere to the general underlying trend, at least to first order. Comparatively nearby objects exhibit negative velocities, whereas distant targets display high positive velocities (i.e., the PN and cluster pair). The magnitude of the velocitydistance gradient is especially pertinent, since it implies that two objects along the sightline that feature similar velocities may be nearly equidistant. In other words, the steep slope indicates that two remote objects sharing comparable velocities are at a common distance. A pronounced gradient likewise mitigates uncertainties arising from imprecise velocity determinations (Table 1). Admittedly, the PN and cluster, in concert with the more remote Cepheids, appear systematically offset from the predicted velocity-distance correlation. However, various uncertainties promulgate into the aforementioned determination (e.g., distance to the Galactic center, Majaess 2010), and deviations from simple Galactic rotation are well known. Andrews-Lindsay 1 is also an older open cluster. Anthropic arguments aside, Fig. 5 provides evidence for an association between PHR 1315-6555 and Andrews-Lindsay 1. In this instance the probability of detecting two unrelated remote objects that exhibit consistent parameters $(r, d, R V)$ is low.

\section{Conclusion}

New multiband $\left(B V J H W_{1-4}\right)$ observations for Andrews-Lindsay 1 and the surrounding field were analyzed, with the objective of highlighting the correct set of cluster parameters (Table 1) and bolstering the link to PHR 1315-6555. Red clump stars identified in near-infrared photometry were exploited to constrain the run of reddening with distance for the $\ell \sim 305^{\circ}$ sightline (Fig. 1). Those data were supplemented with information gleaned from Cepheid variables. The observations imply that the bulk of the reddening is foreground to Andrews-Lindsay 1 and is characterized by $E(J-H)=0.24 \pm 0.03$. The reddening along $\ell \sim 305^{\circ}$ increases in concert with distance until $\sim 4 \mathrm{kpc}$, and thereafter remains nearly constant (Figs. 1, 5). The corresponding optical color excess matches that cited for the PN (Frew 2008; Parker et al. 2011) to within the uncertainties (Table 1).

The distance for Andrews-Lindsay 1 was inferred from a solar isochrone fit to cluster stars in color-magnitude $(B V J H)$ diagrams (Figs. 2 and $4, d_{J H}=9.9 \pm 0.6 \mathrm{kpc}, d_{B V}=10.1 \pm$ $0.5 \mathrm{kpc}$, and $\log \tau=8.90 \pm 0.15)$. The mean distance derived lies between the Janes \& Phelps (1994) and Carraro \& Munari (2004) estimates. A velocity-distance correlation predicted for $\ell \sim 305^{\circ}$ from Galactic rotation generally agrees with the empirical trend delineated by Cepheids, PHR 1315-6555, and Andrews-Lindsay 1. Specifically, the first exhibit negative velocities conducive to nearer targets, whereas the last two phenomena feature high positive velocities tied to distant objects (Fig. 5). The steep correlation indicates that remote objects that are kinematically and spatially coincident along $\ell \sim 305^{\circ}$ are likely associated. The suite of evidence favors an association between the PN PHR 1315-6555 and the open cluster Andrews-Lindsay 1 (Parker et al. 2006, 2011; Majaess et al. 2007; Frew 2008).

Acknowledgements. D.M. is grateful to the following individuals and consortia whose efforts, advice, or encouragement enabled the research: G. Jacoby, D. Frew, A. Parker, P. Frinchaboy, 2MASS, P. Stetson (DAOPHOT), G. Cibis, F. van Leeuwen, F. Benedict, L. Kiss, W. Gieren, D. Balam, B. Skiff, L. Gallo, R. Thacker, Webda (E. Paunzen), W. Dias, CDS (F. Ochsenbein, T. Boch, P. Fernique), arXiv, and NASA ADS. This publication makes use of data products from the Wide-field Infrared Survey Explorer, which is a joint project of the University of California, Los Angeles, and the Jet Propulsion Laboratory/California Institute of Technology, funded by NASA; observations obtained at the Southern Astrophysical Research (SOAR) telescope (program ID: CN2013A-157), which is a joint project of the Ministério da Ciência, Tecnologia, e Inovação (MCTI) da República Federativa do Brasil, the US National Optical Astronomy Observatory (NOAO), the University of North Carolina at Chapel Hill (UNC), and Michigan State University (MSU).

\section{References}

Ahumada, J. A., \& Lapasset, E. 2007, A\&A, 463, 789

Alonso, A., Arribas, S., \& Martinez-Roger, C. 1996, A\&A, 313, 873

Benedict, G. F., McArthur, B. E., Feast, M. W., et al. 2007, AJ, 133, 1810

Benedict, G. F., McArthur, B. E., Napiwotzki, R., et al. 2009, AJ, 138, 1969

Berdnikov, L. N. 2008, VizieR Online Data Catalog: II/285

Berdnikov, L. N., Dambis, A. K., \& Vozyakova, O. V. 2000, A\&AS, 143, 211

Bonatto, C., \& Bica, E. 2007, MNRAS, 377, 1301

Bonatto, C., \& Bica, E. 2010, A\&A, 516, A81

Bonatto, C., \& Bica, E. 2011, MNRAS, 415, 2827

Bonatto, C., Bica, E., \& Girardi, L. 2004, A\&A, 415, 571

Bonatto, C., Bica, E., \& Santos, J. F. C. 2008, MNRAS, 386, 324

Carraro, G., \& Munari, U. 2004, MNRAS, 347, 625

Carraro, G., Vallenari, A., \& Ortolani, S. 1995, A\&A, 300, 128

Carraro, G., Janes, K. A., \& Eastman, J. D. 2005, MNRAS, 364, 179

Carraro, G., Turner, D., Majaess, D., \& Baume, G. 2013, A\&A, 555, A5

De Marco, O., Passy, J.-C., Frew, D. J., Moe, M., \& Jacoby, G. H. 2013, MNRAS, 428, 2118

Frew, D. J. 2008, Ph.D. Thesis

Frinchaboy, P. M., Munoz, R. R., Majewski, S. R., et al. 2004a

[arXiv: astro-ph/0411127]

Frinchaboy, P. M., Majewski, S. R., Crane, J. D., et al. 2004b, ApJ, 602, L21

Giammanco, C., Sale, S. E., Corradi, R. L. M., et al. 2011, A\&A, 525, A58

Girardi, L., Bertelli, G., Bressan, A., et al. 2002, A\&A, 391, 195

Jacoby, G. H., Kronberger, M., Patchick, D., et al. 2010, PASA, 27, 156

Jacoby, G. H., Ciardullo, R., De Marco, O., et al. 2013, ApJ, 769, 10

Janes, K. A., \& Phelps, R. L. 1994, AJ, 108, 1773

Kiss, L. L., Szabó, G. M., Balog, Z., Parker, Q. A., \& Frew, D. J. 2008, MNRAS, 391,399

Landolt, A. U. 1992, AJ, 104, 340 
A\&A 567, A1 (2014)

Lutz, J. H., Braxton, K., McKeever, J., et al. 2012, AAS Meet. Abstracts, 219, 239.03

Majaess, D. 2010, Acta Astron., 60, 55

Majaess, D. J., Turner, D. G., \& Lane, D. J. 2007, PASP, 119, 1349

Majaess, D., Turner, D., \& Lane, D. 2009, Acta Astron., 59, 403

Majaess, D., Turner, D., Moni Bidin, C., et al. 2011a, ApJ, 741, L27

Majaess, D., Turner, D., \& Gieren, W. 2011b, ApJ, 741, L36

Majaess, D. J., Turner, D. G., Lane, D. J., \& Krajci, T. 2011c, J. Am. Assoc.

Variable Star Observers, 39, 219

Majaess, D., Turner, D., Gieren, W., \& Lane, D. 2012a, ApJ, 752, L10

Majaess, D., Turner, D., \& Gieren, W. 2012b, PASP, 124, 1035

Majaess, D., Carraro, G., Moni Bidin, C., et al. 2013, A\&A, 560, A22

Moni Bidin, C., Majaess, D., Bonatto, C., et al. 2014, A\&A, 561, A119

Munari, U., \& Carraro, G. 1996, A\&A, 314, 108

Neckel, T., Klare, G., \& Sarcander, M. 1980, A\&AS, 42, 251

Ngeow, C.-C., Gieren, W., \& Klein, C. 2014, 2014, Proc. IAU Symp., 301, 123

O'dell, C. R. 1963, PASP, 75, 370

Parker, Q. A., Acker, A., Frew, D. J., et al. 2006, MNRAS, 373, 79
Parker, Q. A., Frew, D. J., Miszalski, B., et al. 2011, MNRAS, 413, 1835 Pauls, R., \& Kohoutek, L. 1996, Astron. Nachr., 317, 413

Paunzen, E., \& Netopil, M. 2006, MNRAS, 371, 1641

Pietrukowicz, P., Udalski, A., Soszyński, I., et al. 2012, ApJ, 750, 169

Schlafly, E. F., \& Finkbeiner, D. P. 2011, ApJ, 737, 103

Skiff, B. A. 2013, VizieR Online Data Catalog: II/23

Stanghellini, L., Shaw, R. A., \& Villaver, E. 2008, ApJ, 689, 194

Stetson, P. B. 1987, PASP, 99, 191

Turner, D. G. 2001, Odessa Astronomical Publications, 14, 166

Turner, D. G. 2010, Ap\&SS, 326, 219

Turner, D. G. 2013, CJP, submitted [arXiv: 1310.0014]

Turner, D. G., Rosvick, J. M., Balam, D. D., et al. 2011, PASP, 123, 1249

Turner, D. G., van den Bergh, S., Younger, P. F., et al. 2012, AJ, 144, 187

van Leeuwen, F. 2007, A\&A, 474, 653

van Leeuwen, F. 2009, A\&A, 497, 209

van Leeuwen, F. 2013, A\&A, 550, L3

Wright, E. L., Eisenhardt, P. R. M., Mainzer, A. K., et al. 2010, AJ, 140 1868 